\title{
MPMI ACKNOWLEDGMENT OF REVIEWERS 2019
}

The success of Molecular Plant-Microbe Interactions depends on the quality of manuscripts submitted by authors and on the care and competence with which they are reviewed. It is the policy of the Editorial Board to solicit reviews of manuscripts from specialists most qualified to review them. In addition to members of the Editorial Board, the individuals listed below provided constructive critical reviews of one or more manuscripts during the past year. Their names are published here in grateful appreciation for their contributions to the journal.

\begin{tabular}{|c|c|}
\hline Mine Akira & David Cook \\
\hline Gladys Alexandre & Bret Cooper \\
\hline Juliana Almario & Kevin Cope \\
\hline Jeffrey Anderson & Teresa Coutinho \\
\hline Jonathan Anderson & Daniel Croll \\
\hline Jean-Michel Ané & Fuhao Cui \\
\hline Chris Argueso & Sébastien Cunnac \\
\hline Markus Arnold & Christopher Dardick \\
\hline Adi Avni & Stephanie Daval \\
\hline Guus Bakkeren & Leonardo De La Fuente \\
\hline David Baltrus & Stéphane De Mita \\
\hline Lois Banta & Etienne Delannoy \\
\hline Jeri D. Barak & Pierre-Marc Delaux \\
\hline Paola Barba Burgos & Yinyue Deng \\
\hline Rebecca Bart & George diCenzo \\
\hline Jacques Batut & Rebecca Dickstein \\
\hline Eduardo Bejarano & María Diéguez \\
\hline Richard Bélanger & Pingtao Ding \\
\hline Lassaad Belbahri & Elke Dittmann \\
\hline Gilles Bena & Peter Dodds \\
\hline Patricia Bernal & Suomeng Dong \\
\hline Alison Berry & Nicole Donofrio \\
\hline Richard Berthome & Daolong Dou \\
\hline Carmen Bianco & J. Maxwell Dow \\
\hline Laurence Bindschedler & Liquan Du \\
\hline Adam Bogdanove & John Dunbar \\
\hline Melvin Bolton & Sebastien Duplessis \\
\hline Freddy Boutrot & Owain Edwards \\
\hline Joanna Bowen & Malin Elfstrand \\
\hline Veronique Brault & Courtney Ellison \\
\hline Daren Brown & Manuel Espinosa-Urgel \\
\hline Sigal Brown & Thomas Eulgem \\
\hline Liliana Cano & Katherine Evans \\
\hline Tomas Canto & Dominique Expert \\
\hline Delphine Capela & Johannes Fahrentrapp \\
\hline Jeffrey Caplan & Jiaqin Fan \\
\hline Massimiliano Cardinale & Xie Fang \\
\hline John Carr & Yufeng Fang \\
\hline Clare Casteel & Franco Faretra \\
\hline Eunyoung Chae & Francesco Favaron \\
\hline Jeff Chang & Judith Felten \\
\hline Trevor Charles & Baomin Feng \\
\hline Subhadeep Chatterjee & Turlough Finan \\
\hline Gong-You Chen & Hans-Martin Fischer \\
\hline Xiao-Ren Chen & Victor Flors \\
\hline Xuewei Chen & Svetlana Folimonova \\
\hline Yet-Ran Chen & Vincent Fondong \\
\hline Chiu-Ping Cheng & Jose Fonseca \\
\hline Haiping Cheng & Elizabeth Fontes \\
\hline Jiasen Cheng & Michael Freitag \\
\hline Xiaofei Cheng & Timothy Friesen \\
\hline Shawn Christensen & Zhengqing Fu \\
\hline Nicoletta Contaldo & Dean Gabriel \\
\hline
\end{tabular}

Daniel Gage
Jean-Luc Gallois
Elena Ganusova
Mengsheng Gao
Matteo Garbelotto
María Garcia-Pedrajas
Donald Gardiner
Stanton Gelvin
Eric Giraud
Pierre Gladieux
Stefanie Gläser
Michael Göttert
Benjamin Gourion
Peter Gresshoff
Niklaus Grünwald
Biao Gu
Michelle Guo
Prasad Gyaneshwar
Dennis Halterman
John Hammond
Sang-Wook Han
Cara Haney
Anton Hartmann
Ishtiaq Hassan
Makoto Hayashi
Jin He
Jason Heindl
Rosa Hermosa
Shuguo Hou
Yiping Hou
Chuan-Chih Hsu
Li Huang
Lin Huang
Edgar Huitema
Michael Hynes
Hyong Woo Choi
Yuki Ichinose
Alexander Idnurm
Juan Imperial
Roger Innes
Solmaz Irani
Richard Jacoby
Sylwia Jafra
Mukesh Jain
Euan James
Monika Janczarek
Katrin Janik
Sunhyn Jeon Jing Joosten

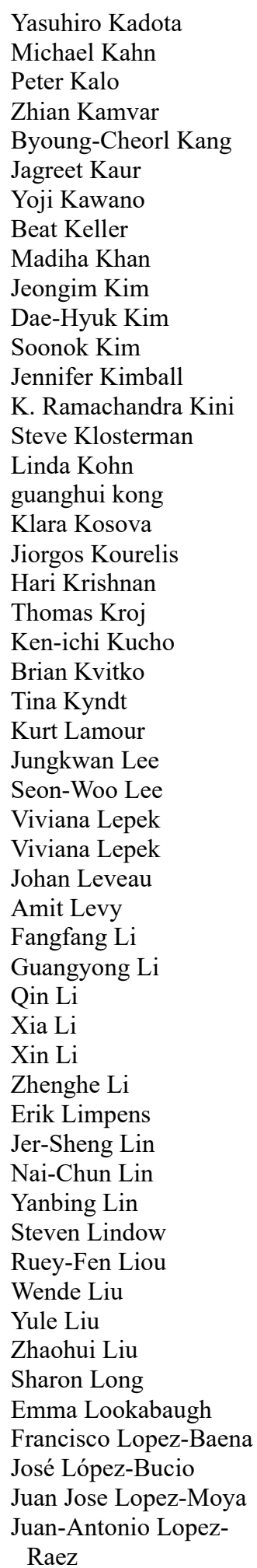


Jennifer Lorang

Rosa Lozano-Durán

Dongping Lu

Yushi Luan

Ole Lund

Li Luo

Lijun Ma

Zhenchuan Ma

Alberto Macho

Robert Malinowski

John W. Mansfield

Sophie Mantelin

Ramona Marasco

Katharina Markmann

Roland Marmeisse

Juan-Francisco Martín

Pedro Martinez-Gomez

Esperanza Martínez-

Romero

Florentine Marx

Cristina Marzachi

Chikara Masuta

Ulrike Mathesius

Hidenori Matsui

Ronan Mccarthy

John McDowell

Julien Mercier

Richard Michelmore

Wolfgang Moeder

Jacqueline Monaghan

Jorge Mondego

Paula Moolhuijzen

Christina Moon

Hiromitsu Moriyama

Paul F. Morris

Gary Muehlbauer

Raj Mujumdar

Arijit Mukherjee

Luis Mur

Jeremy Murray

Rita Musetti

Paul Nabity

Marina Nadal

Hideo Nakashita

Shigetou Namba

Ashis Nandi

Jesús Navas-Castillo

Andrew Nelson

Eric Newberry

Arvin Nickzad

Yuese Ning

Yulong Niu

Noor Khan Noor Khan
Richard O'Connell

Aleksandra Obrępalska-

Stęplowska

Kerry ODonnell

Chang-Sik Oh

Marc Ongena

Hongyu Pan

Huairong Pan

Daniel Panaccione

Francesco Paolocci

Veronico Pasqua

Luciane Passaglia

Katharina Pawlowski

Nemo Peeters

Edel Pérez-López

Sambasivam Periyannan

Michael Perlin

Xavier Perret

Minna Pirhonen

Catalina Pislariu

Thomas Platt

Jonathan Plett

Neha Potnis

Nadine Präg

Robert Proctor

Guo-Liang Qian

Yongli Qiao

Ling Qing

Feng Qu

Shen Chun Qu

Carmen Quinto

Lucía Ramírez

Katrina Ramonell

ALN Rao

Uma Rao

Regina Redman

Luis Rey

Jane Robb

Jeffrey Rollins

Cristina Rosa

Jason Rudd

Soledad Sacristan

M.A. Saghai Maroof

Yusuke Saijo

Blanca San Segundo

Andrea Sanchez-Vallet

Helene Sanfacon

Juan Sanjuan

Christopher Schardl

Birgit Scharf

Jim Schoelz

Hilke Schröder

Steve Scofield
C. Segonzac

Michael Seidl

Shigemi Seo

Shankar Shakya

Wei-Xing Shan

Danyu Shen

Won-Bo Shim

Ken Shirasu

Esther Singer

Rajarammohan

Sivasubramanian

Nicholas Skandalis

Geert Smant

Hokyoung Son

María José Soto

Elineide Souza

Gary Stacey

Christian Staehelin

Remco Stam

Drake Stenger

Jane Stewart

Wolfgang Stöggl

Giannis Stringlis

Senthil Subramanian

Jianbin Sun

Nobuhiro Suzuki

Raffaele sylvain

Michael Taliansky

James Tambong

Xiaorong Tao

Satyanarayana Tatineni

Ryohei Terauchi

Hans Thordal-

Christensen

Zhendong Tian

Jens Tilsner

Louis Tisa

Sotirios Tjamos

Lam-Son Phan Tran

Diwaker Tripathi

Sucheta Tripathy

Eric Triplett

Alexandre Tromas

Kenichi Tsuda

Brett Tyler

Marc Valls

Vittorio Venturi

Satish K. Verma

Ramesh Vetukuri

Muriel Viaud

Vinod Vijayakumar

Antonia Vincent

Corina Vlot
Guan-Feng Wang

Guo-Liang Wang

Nian Wang

Ruoyu Wang

Tao Wang

Xian-Bing Wang

Xiaofeng Wang

Xiaojie Wang

Xiaowei Wang

Xiue Wang

Zonghua Wang

Taiyun Wei

Yangdou Wei

Anna Whitfield

John Whitney

Richard Wilson

Kathrin Wippel

Lennart Wirthmueller

Katja Witzel

Charles Woloshuk

Koonho Wong

Sek-Man Wong

Hanhong Xu

Xiangming $\mathrm{Xu}$

Jun-Yi Yang

Li Yang

Qin Yang

Xiuling Yang

Jian Yao

Wenwu Ye

Yonghao Ye

Wenbing Yin

Zhongchao Yin

Kentaro Yoshida

Hirofumi Yoshioka

Carolyn Young

Diqiu Yu

Hongli Yuan

Xiaochen Yuan

Madiha Zaynab

Chunquan Zhang

Qiong Zhang

Ruifu Zhang

Xiaoming Zhang

Yong Zhang

Young Zhang

Zhengguang Zhang

Bingyu Zhao

Zhijian Zhao

Xueping Zhou

Keyan Zhu-Salzman

Veronique Ziegler-Graff

Sabine Zimmermann 